\title{
Antitumor activity of everolimus in recurrent metastatic endometrial cancer with PTEN deletion: a case report
}

\author{
Xuhong Zhang ${ }^{1}$, Junping Shi ${ }^{2}$ Junying $\mathrm{Li}^{2}$, Xiaoliang Shi ${ }^{2}$ Xiaoliang Liu ${ }^{2}$, Yingli Zhang ${ }^{3}$, Yanzhi Cui ${ }^{4}$ \\ ${ }^{1}$ Gynecology Department, Tianjin Department Medical University General Hospital, Tianjin, China; ${ }^{2}$ OrigiMed Co. Ltd, Shanghai, China; \\ ${ }^{3}$ Department of Gynecologic Oncology, Zhejiang Cancer Hospital, Hangzhou, China; ${ }^{4}$ Department of Medical Oncology, the Fourth Hospital of \\ Hebei Medical University, Shijiazhuang, China \\ Correspondence to: Yanzhi Cui. Department of Medical Oncology, The Fourth Hospital of Hebei Medical University, 12 Health Road, Shijiazhuang \\ 050011, China. Email: cyz_2005@126.com.
}

\begin{abstract}
Endometrial cancer (EC) is one of the most common gynecological tumors. The first-line treatment for advanced EC is chemoradiotherapy. However, patients in poor health, such as those with intestinal obstruction, have limited tolerance for the side effects of chemoradiotherapy. Individualized precision treatment may bring new hope to these patients. Herein, we have reported on a 56-year-old female patient with metastatic EC combined with severe intestinal obstruction. Due to her inability to tolerate needle biopsy and standard treatment protocols, next-generation sequencing (NGS)-based circulating tumor DNA (ctDNA) testing was performed and PTEN deletion was found. Following, the patient commenced everolimus $(10 \mathrm{mg}, \mathrm{qd})$ treatment and partial shrinkage of metastases was observed one month later. Then, everolimus (10 mg, qd) plus carboplatin (100 mg d1, 8, 15, q28d) for 2 cycles, everolimus (10 mg, qd) plus carboplatin (200 mg d1, 8, q21d) for 2 cycles, and everolimus (10 mg, qd) plus carboplatin (200 mg d1, 2, q21d) for 2 cycles were performed, and the patient got partial response for 10 months. From June 2019, the patient continued to benefit from everolimus and subsequently experienced continued benefit for more than 12 months. This is the first reported case of an EC patient who benefited from everolimus as a firstline treatment based on PTEN deletion. This case provides important clinical experience for the precision treatment of patients with advanced EC.
\end{abstract}

Keywords: Endometrial cancer (EC); circulating tumor DNA (ctDNA); PTEN deletion; everolimus; case report

Submitted Jun 04, 2021. Accepted for publication Aug 19, 2021.

doi: 10.21037 /gs-21-422

View this article at: https://dx.doi.org/10.21037/gs-21-422

\section{Introduction}

Endometrial carcinoma (EC) is the fourth most commonly diagnosed tumor and the fourth leading cause of death among females in the world (1). The incidence rate of the EC is about 20 to 30 per10 million people, and is increasing year by year (2). The poor prognosis of recurrence EC is partly due to the poor responses to salvage chemotherapy and limited treatment options (3). Alternative therapeutic options are desperately needed.

Mutation of the PI3K/AKT/mTOR pathway is common in EC, and most of them involve the loss of PTEN (4). PTEN regulates the activity of $\mathrm{PI} 3 \mathrm{~K} / \mathrm{AKT} / \mathrm{mTOR}$ pathway through phosphorylation, which plays an important role in the development and maintenance of endometrial cancer (5). A number of studies have evaluated the role of single-agent mTOR inhibition in recurrent EC as both primary and second-line therapy (6-8). Although the remission rate of single drug therapy is not ideal, blocking this biological pathway is a reasonable way to control the disease. Everolimus is an effective, selective and orally active mTOR inhibitor (9). In recent years, everolimus combined with hormonal therapy was reported to be effective in recurrent endometrioid EC patients $(10,11)$. These studies suggest that mTOR inhibitors such as everolimus may effectively alleviate the resistance of endocrine therapy. 
Molecular analysis of tumor tissue has been widely applied to the treatment of solid malignant tumor patients. However, whether there are enough samples is a concern for tumor tissue-based testing (12). Due to the heterogeneity of tumor cells, tissue obtained from primary surgical resection or biopsy may not reflect the current tumor molecular composition. While plasma-derived circulating tumor DNA (ctDNA) provides a more convenient and real-time option to detect the potentially actionable mutations of tumor. So far, the U.S Food and Drug Administration (FDA) has approved a number of ctDNA testing for many cancers in progression on or after endocrine therapy (13). In this case report, we present a case of recurrent metastatic EC with PTEN deletion. To our knowledge, this is the first report of PTEN deletion identified by ctDNA testing in an EC patient. The evaluation of everolimus in patients with PTEN deficiency will provide evidence for future precision treatment of ECs. We present the following article in accordance with the CARE reporting checklist (available at https://dx.doi.org/10.21037/gs-21-422).

\section{Case presentation}

A 56-year-old postmenopausal female presented with abnormal vaginal bleeding in November 2012. Magnetic resonance imaging (MRI) examination revealed uneven endometrial signals and abnormal signals in the left posterior wall of the uterus, suggesting uterine fibroids (Figure 1A). The patient received diagnostic curettage, and the pathology result indicated EC. She underwent complete surgical staging including hysterectomy, bilateral salpingo-oophorectomy, and bilateral pelvic and paraaortic lymphadenectomy. The pathologic result showed a well-differentiated polypoid adenocarcinoma of the endometrium. The tumor was $2 \mathrm{~cm} \times 1.6 \mathrm{~cm}$, without any metastasis, indicating the International Federation of Gynecology and Obstetrics (FIGO) stage IA. At the conclusion of the case there was no evidence of gross residual disease. The immunohistochemistry (IHC) results were: estrogen receptor (ER) (++), progesterone receptor (PR) (+), human epidermal growth factor receptor 2 (HER-2) (-), Ki-67 (+), and p53 (-). The patient had been disease-free until October 2017, when she developed intestinal obstruction and epigastric distention with anorexia, nausea, constipation, and weight loss of $15 \mathrm{~kg}$ within 3 months. The computed tomography (CT) examination showed multiple metastatic lesions in the liver, abdominal, and pelvic cavity in January 2018
(Figure 1B). Abdominal pelvic fluid was also revealed by CT. The levels of cancer antigen 125 (CA125), CA199, and carcinoembryonic antigen (CEA) were $2,726 \mathrm{U} / \mathrm{mL}$ (reference value: $0-35 \mathrm{U} / \mathrm{mL}$ ), $220.5 \mathrm{U} / \mathrm{mL}$ (reference value: $0-35 \mathrm{U} / \mathrm{mL}$ ), and $10.33 \mathrm{ng} / \mathrm{mL}$ (reference value: $0-5 \mathrm{ng} / \mathrm{mL}$ ), respectively. She was in a poor general condition with a performance status (PS) score of 3 .

The patient vehemently refused to undergo puncture for further diagnosis after referral to our center. Due to severe intestinal obstruction and extreme gastrointestinal side effects, the patient was unable to receive a singleagent chemotherapeutic of paclitaxel or the combination of paclitaxel and carboplatin. The patient voluntarily accepted the next-generation sequencing (NGS) based ctDNA testing which was performed by OrigiMed (Shanghai, China), from where the laboratory was certified by the College of American Pathologists (CAP) and Clinical Laboratory Improvement Amendments (CLIA). Considering the detected PTEN deletion, the patient commenced everolimus (10 mg, qd) treatment on 15 January 2018. On 15 February 2018, the patient's symptoms were significantly relieved. The CT imaging showed partial shrinkage of metastases and indicated a stable disease (SD) (Figure 1C). The patient was then treated with everolimus (10 mg, qd) plus carboplatin (100 mg d1, 8, 15, q28d) for 2 cycles, everolimus $(10 \mathrm{mg}$, qd) plus carboplatin (200 mg d1, 8, q21d) for 2 cycles, and everolimus (10 mg, qd) plus carboplatin (200 mg d1, 2, q21d) for 2 cycles. The clinical efficacy was evaluated as partial response (PR) by CT imaging in July 2018 (Figure 1D). From July 2018, the patient received everolimus (10 mg, qd) monotherapy, and the clinical efficacy was evaluated as SD by CT imaging in January 2019 (Figure 1E). From June 2019, the patient continued to benefit from everolimus. During the treatment, the patient had no special discomfort or side effects. The patient had a history of hypertension, and took oral nifedipine to control blood her pressure.

The PTEN c.36_79+4del deletion and PIK3R1 c.130072_1426-301delinsCT mutation of this patient detected by NGS-based ctDNA detection are shown in Figure 2.

The authors are accountable for all aspects of the work in ensuring that questions related to the accuracy or integrity of any part of the work are appropriately investigated and resolved. All procedures were in accordance with the ethical standards of the responsible committee on human experimentation (institutional and national) and with the Helsinki Declaration (as revised in 2013). Written informed consent was obtained from the patient for publication of this 


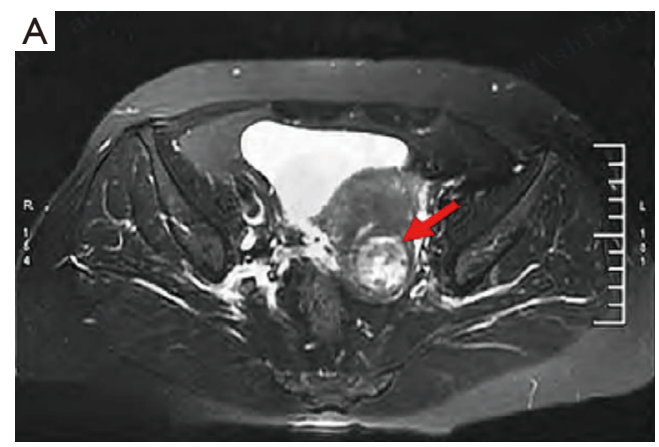

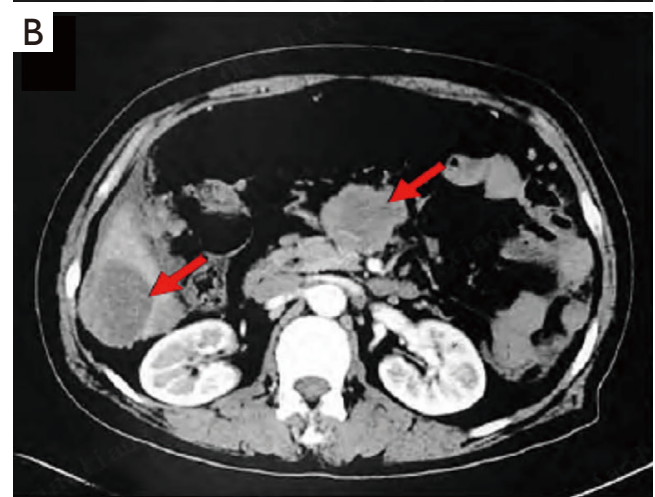

Baseline

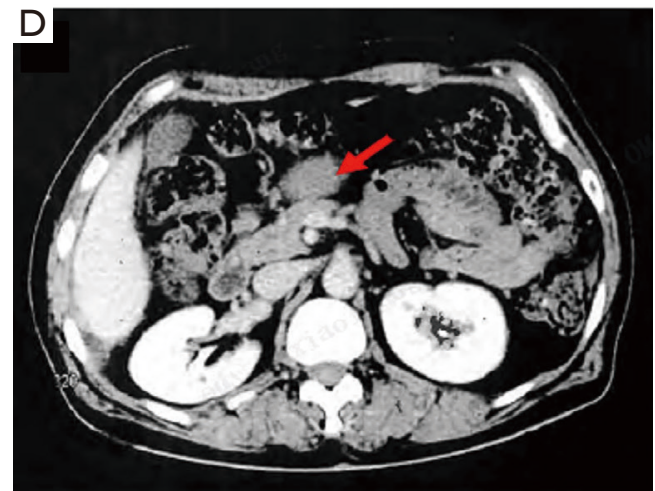

Six months after Everolimus treatment

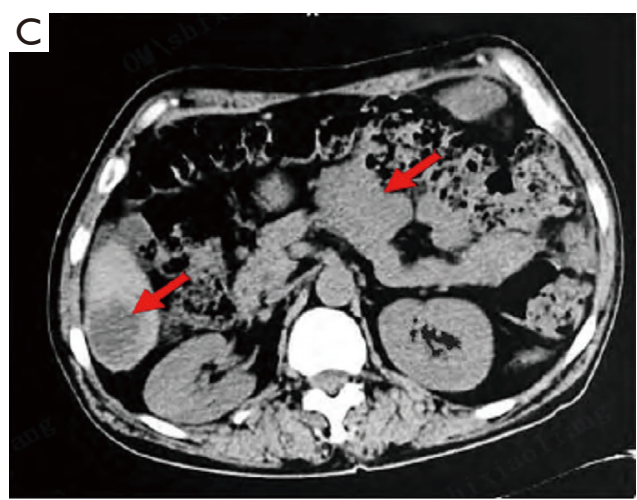

One month after Everolimus treatment

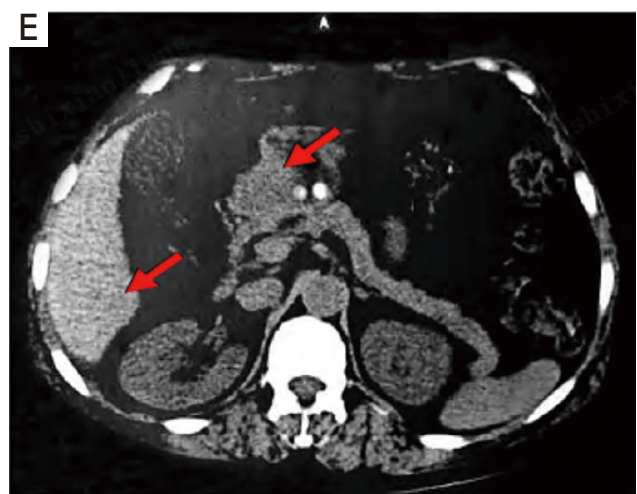

Twelve months after Everolimus treatment

Figure 1 Image evaluation of the patient. (A) MRI examination in December 2012. CT scans, (B) baseline; (C) the patient received everolimus treatment for 1 month; the patient recieved everolimus plus carboplatin treatment for 6 months (D), and 12 months (E). The red arrow indicates the location of the tumor. MRI, magnetic resonance imaging; CT, computed tomography.

case report and accompanying images. A copy of the written consent is available for review by the editorial office of this journal. The study was approved by the Ethics Committee of the Fourth Hospital of Hebei Medical University.

\section{Discussion}

Liquid biopsy, which include sampling of blood and other body fluids, is becoming to be effective for cancer diagnosis, routine monitoring, and prognosis. The ctDNA is a popular liquid biopsy biomarker that has been found to be easily detectable in the plasma of cancer patients (14). At present, an increasing number of studies have shown that ctDNA detection has outstanding clinical application value in monitoring tumor progression and precision therapy $(13,15)$. CtDNA detection is a non-invasive and easily accessible way to determine prognosis and treatment methods (13). However, tissue biopsy is still the gold 


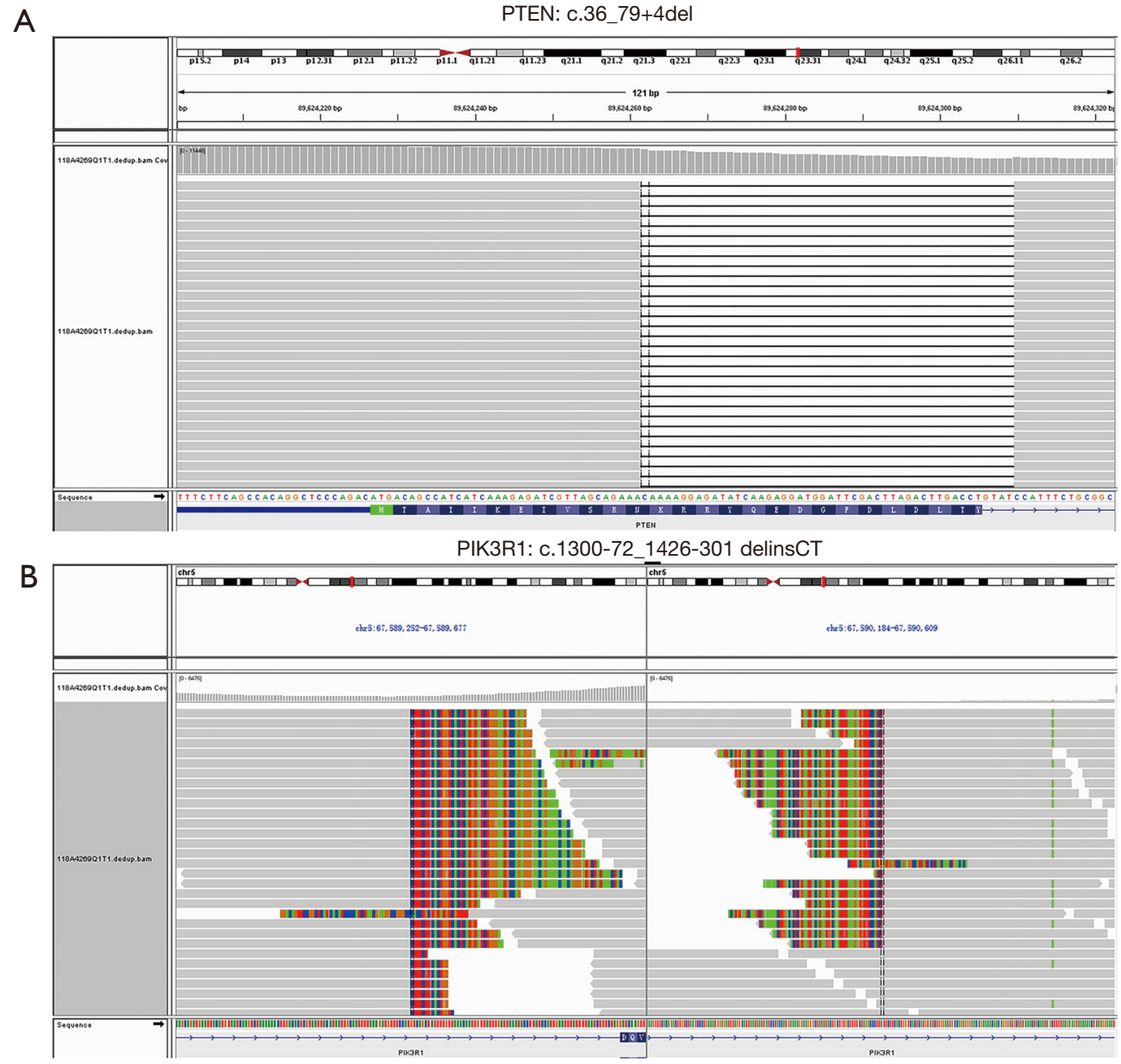

Figure 2 The integrative genomics viewer snapshot of mutations detected by NGS-based ctDNA detection. (A) PTEN c.36_79+4del deletion mutation; (B) PIK3R1 c.1300-72_1426-301delinsCT mutation.

standard for gene detection, with higher accuracy and specificity. The use of ctDNA is a good alternative when tissue is not available. It can also provide some certain references for clinical diagnosis and treatment. In clinical practice, reference is much better and meaningful than no reference. The patient in our study had a poor physical condition with intestinal obstruction during tumor recurrence and had strongly opposed invasive diagnosis and traditional treatment, including needle biopsy, and traditional radiotherapy and chemotherapy. The use of ctDNA detection provided an option for diagnosis and treatment of this patient.

Through NGS-based ctDNA testing, PTEN deletion and PIK3R1 mutation were identified in this patient. The $P T E N$ gene is the most significantly altered gene (77\%) and the mutation frequency of PIK3R1 is about $15 \%$ in EC (16).
However, there are no FDA approved anti-tumor drugs targeting PTEN or PIK3R1. The PTEN gene is a negative regulator of the PI3K-AKT-mTOR pathway, which plays an important role in regulating of cell growth and survival (17). Both PTEN deletion and PIK3R1 mutations can activate PI3K activity and promote phosphorylation of AKT (17), suggesting that Akt inhibitors or mTOR inhibitors may be potential therapies for tumors harboring these gene mutations. Several studies have evaluated the effect of single-agent mTOR inhibitors in recurrent ECs as both first- and second-line therapy (6-8). Although the objective effective rate was 0-24\%, the stable SD rates have reached as high as $90 \%$.

Everolimus is a rapamycin (mTOR) inhibitor that has an antitumor effect on cancers $(18,19)$. Everolimus effectively reduced endometrial proliferations progression of and thyroid hyperplasia in PTEN-deficient mouse models (20). 
The phase II trial of GINECO demonstrated efficacy and acceptable tolerability of everolimus in advanced or metastatic EC patients with chemotherapy-refractory, which supported the further development of PI3K-targeted therapies in EC (21). In addition, everolimus combined with letrozole in patients with advanced and recurrent EC showed a clinical benefit rate of $40 \%$ and objective response rate of $32 \%$ among 35 evaluable patients, respectively (11). Epidermal growth factor receptor 2-positive breast cancer with PIK3CA mutations, PTEN loss, or hyperactive PI3K pathway may have opportunity to benefit from everolimus (22). The phase II trial (NCT02029001) of the mTOR inhibitor everolimus for the treatment of patients with advanced tumors carrying PIK3R1 mutations is ongoing (23). In addition, it was reported that a patient with $P T E N$-deficient metastatic endometrioid endometrial adenocarcinoma displayed a profound sensitivity to platinum-containing regimens (24). In this study, the patient was treated with everolimus for first-line treatment and achieved a certain effect and her physical condition improved significantly. After the addition of carboplatin chemotherapy on the basis of everolimus, the mass was further reduced, and the therapeutic effect was evaluated as PR. The conventional treatment of endometrial cancer includes surgery, radiotherapy, chemotherapy, and hormone therapy. Slomovitz et al. shows that everolimus and letrozole may be a reasonable choice for second-line treatment of recurrent endometrioid EC (25). Combined with this case, especially for patients with advanced or recurrent EC, we suggest that NGS based genomic detection is necessary for the identification of potential drug targets. For patients with PETN, everolimus combine with carboplatin chemotherapy may be a potential choice.

\section{Conclusions}

We have presented a metastatic EC patient with PTEN deletion who achieved PR after receiving the mTOR inhibitor everolimus, and benefited from it for more than 12 months. To our knowledge, this was the first report of patients with advanced EC for targeted therapy through NGS-based ctDNA testing. This case supports the possibility of everolimus combined with carboplatin chemotherapy in the treatment of EC patients with PTEN mutation, and provides important clinical experience for the precision treatment of patients with advanced ECs.

\section{Acknowledgments}

Funding: This work was supported by National Natural Science Foundation of China (81602267).

\section{Footnote}

Reporting Checklist: The authors have completed the CARE reporting checklist. Available at https://dx.doi. org/10.21037/gs-21-422

Conflicts of Interest: All authors have completed the ICMJE uniform disclosure form (available at https://dx.doi. org/10.21037/gs-21-422). All authors have declared no conflicts of interest.

Ethical Statement: The authors are accountable for all aspects of the work in ensuring that questions related to the accuracy or integrity of any part of the work are appropriately investigated and resolved. All procedures were in accordance with the ethical standards of the responsible committee on human experimentation (institutional and national) and with the Helsinki Declaration (as revised in 2013). Written informed consent was obtained from the patient for publication of this case report and accompanying images. A copy of the written consent is available for review by the editorial office of this journal. The study was approved by the Ethics Committee of the Fourth Hospital of Hebei Medical University.

Open Access Statement: This is an Open Access article distributed in accordance with the Creative Commons Attribution-NonCommercial-NoDerivs 4.0 International License (CC BY-NC-ND 4.0), which permits the noncommercial replication and distribution of the article with the strict proviso that no changes or edits are made and the original work is properly cited (including links to both the formal publication through the relevant DOI and the license). See: https://creativecommons.org/licenses/by-nc-nd/4.0/.

\section{References}

1. Bray F, Ferlay J, Soerjomataram I, et al. Global cancer statistics 2018: GLOBOCAN estimates of incidence and mortality worldwide for 36 cancers in 185 countries. CA Cancer J Clin 2018;68:394-424. 
2. Lu KH, Broaddus RR. Endometrial Cancer. N Engl J Med 2020;383:2053-64.

3. Huijgens AN, Mertens HJ. Factors predicting recurrent endometrial cancer. Facts Views Vis Obgyn 2013;5:179-86.

4. Slomovitz BM, Coleman RL. The PI3K/AKT/mTOR pathway as a therapeutic target in endometrial cancer. Clin Cancer Res 2012;18:5856-64.

5. Weigelt $\mathrm{B}$, Warne PH, Lambros MB, et al. PI3K pathway dependencies in endometrioid endometrial cancer cell lines. Clin Cancer Res 2013;19:3533-44.

6. Slomovitz BM, Lu KH, Johnston T, et al. A phase 2 study of the oral mammalian target of rapamycin inhibitor, everolimus, in patients with recurrent endometrial carcinoma. Cancer 2010;116:5415-9.

7. Colombo N, McMeekin DS, Schwartz PE, et al. Ridaforolimus as a single agent in advanced endometrial cancer: results of a single-arm, phase 2 trial. Br J Cancer 2013;108:1021-6.

8. Oza AM, Elit L, Tsao MS, et al. Phase II study of temsirolimus in women with recurrent or metastatic endometrial cancer: a trial of the NCIC Clinical Trials Group. J Clin Oncol 2011;29:3278-85.

9. Hasskarl J. Everolimus. Recent Results Cancer Res 2018;211:101-23.

10. Soliman PT, Westin SN, Iglesias DA, et al. Everolimus, Letrozole, and Metformin in Women with Advanced or Recurrent Endometrioid Endometrial Cancer: A MultiCenter, Single Arm, Phase II Study. Clin Cancer Res 2020;26:581-7.

11. Slomovitz BM, Jiang Y, Yates MS, et al. Phase II study of everolimus and letrozole in patients with recurrent endometrial carcinoma. J Clin Oncol 2015;33:930-6.

12. Shih JY, Gow CH, Yu CJ, et al. Epidermal growth factor receptor mutations in needle biopsy/aspiration samples predict response to gefitinib therapy and survival of patients with advanced nonsmall cell lung cancer. Int $\mathbf{J}$ Cancer 2006;118:963-9.

13. Moss EL, Gorsia DN, Collins A, et al. Utility of Circulating Tumor DNA for Detection and Monitoring of Endometrial Cancer Recurrence and Progression. Cancers (Basel) 2020;12:2231.

14. Alix-Panabières C, Pantel K. Clinical Applications of Circulating Tumor Cells and Circulating Tumor DNA as Liquid Biopsy. Cancer Discov 2016;6:479-91.

15. De Rubis G, Krishnan SR, Bebawy M. Circulating tumor DNA - Current state of play and future perspectives. Pharmacol Res 2018;136:35-44.

16. Brooks RA, Fleming GF, Lastra RR, et al. Current recommendations and recent progress in endometrial cancer. CA Cancer J Clin 2019;69:258-79.

17. Hollander MC, Blumenthal GM, Dennis PA. PTEN loss in the continuum of common cancers, rare syndromes and mouse models. Nat Rev Cancer 2011;11:289-301.

18. Hortobagyi GN, Chen D, Piccart M, et al. Correlative Analysis of Genetic Alterations and Everolimus Benefit in Hormone Receptor-Positive, Human Epidermal Growth Factor Receptor 2-Negative Advanced Breast Cancer: Results From BOLERO-2. J Clin Oncol 2016;34:419-26.

19. Motzer RJ, Escudier B, Oudard S, et al. Efficacy of everolimus in advanced renal cell carcinoma: a doubleblind, randomised, placebo-controlled phase III trial. Lancet 2008;372:449-56.

20. Mirantes C, Eritja N, Dosil MA, et al. An inducible knockout mouse to model the cell-autonomous role of PTEN in initiating endometrial, prostate and thyroid neoplasias. Dis Model Mech 2013;6:710-20.

21. Ray-Coquard I, Favier L, Weber B, et al. Everolimus as second- or third-line treatment of advanced endometrial cancer: ENDORAD, a phase II trial of GINECO. Br J Cancer 2013;108:1771-7.

22. André F, Hurvitz S, Fasolo A, et al. Molecular Alterations and Everolimus Efficacy in Human Epidermal Growth Factor Receptor 2-Overexpressing Metastatic Breast Cancers: Combined Exploratory Biomarker Analysis From BOLERO-1 and BOLERO-3. J Clin Oncol 2016;34:2115-24.

23. Toulmonde M, Le Tourneau C, Italiano A, et al. A randomized, open-label, Phase II trial evaluating the clinical benefit of a maintenance treatment targeting tumor molecular alterations in patients with advanced solid tumors. J Clin Oncol 2015;33:TPS2622.

24. Forster MD, Dedes KJ, Sandhu S, et al. Treatment with olaparib in a patient with PTEN-deficient endometrioid endometrial cancer. Nat Rev Clin Oncol 2011;8:302-6.

25. Slomovitz BM, Filiaci VL, Coleman RL, et al. GOG 3007, a randomized phase II (RP2) trial of everolimus and letrozole (EL) or hormonal therapy (medroxyprogesterone acetate/tamoxifen, PT) in women with advanced, persistent or recurrent endometrial carcinoma (EC): A GOG Foundation study. Gynecologic Oncology 2018;149:2.

Cite this article as: Zhang X, Shi J, Li J, Shi X, Liu X, Zhang Y, Cui Y. Antitumor activity of everolimus in recurrent metastatic endometrial cancer with PTEN deletion: a case report. Gland Surg 2021;10(8):2585-2590. doi: 10.21037/gs-21-422 